\title{
Cross-layer Routing Optimization for Wireless Networks with Cooperative Diversity
}

\author{
Zhiguo Ding and Kin K. Leung \\ Department of Electrical and Electronic Engineering \\ Imperial College London, UK. \\ Email: \{zhiguo.ding, kin.leung\}@imperial.ac.uk
}

\begin{abstract}
In this paper, we study the impact of cooperative transmission on the routing decision for wireless ad-hoc networks. The influence of cooperative transmission to the wireless link cost is first studied at the physical layer. Then the problem of routing optimization is investigated to understand the effects of improved link cost on the routing decision, where the closed-form solution of the optimization problem is developed and later used as a quantitative criterion of the route selection. Our developed analytical and simulation results show that the criteria using cooperative transmission typically yield more efficient routes compared with the non-cooperative schemes.
\end{abstract}

\section{INTRODUCTION}

Future wireless communication networks are expected to support the mixture of real-time applications, such as voice and multimedia teleconferencing, and non-real-time data applications, such as web browsing, messaging and file transfers. Compared with wired environments, the associated communication channels and traffic patterns in mobile wireless networks are more unpredictable. Hence all of these applications impose stringent and diversified Quality of Service (QoS) requirements, which cannot be satisfactorily addressed through the traditional layering network-protocol architecture. Correspondingly, there has recently been increased interest in protocols for wireless networks to exploit the significant interactions between various layers of the protocol stack for performance enhancements. And it has been shown that these cross-layer designs and protocols could be essential for wireless ad-hoc and sensor networks where unpredictable variables such as node mobility, node density and network dimensions make the diverse and stringent wireless QoS requirements difficult to satisfy.

Due to the unreliability of wireless links, it has been of interest to study the impacts of physical-layer techniques on the design of upper-layers, including medium access control (MAC), packet scheduling, power control, routing, transport protocol, and ultimately the QoS at the application level in wireless networks. Opportunistic scheduling could be seen as one of successful examples of cross-layer design, where

Research was sponsored by US Army Research laboratory and the UK Ministry of Defence and was accomplished under Agreement Number W911NF06-3-0001. The views and conclusions contained in this document are those of the authors and should not be interpreted as representing the official policies, either expressed or implied, of the US Army Research Laboratory, the U.S. Government, the UK Ministry of Defense, or the UK Government. The US and UK Governments are authorized to reproduce and distribute reprints for Government purposes notwithstanding any copyright notation hereon. scheduling protocols are designed by taking advantage of the knowledge of wireless link conditions [1], [2]. Among many candidates of physical-layer techniques, multiple-input multiple-output (MIMO) has received significant attention, which can provide spatial diversity and hence represents a powerful technique for interference mitigation and reduction [3], [4]. Cooperative communication provides an alternative way to achieve spatial diversity, where single-antenna terminals in a multiple-user environment share their antennas and form a cluster to assist each other with their data transmission [5], [6]. In [7] and [8] the authors study the cross-layer routing protocol design for energy-constrained networks, where the cooperative transmission technique is used to form a virtual antenna array. It is shown in [7] and [8] that the proposed protocol could yield a different route compared with traditionally non-cross-layer protocols if the circuit processing energy is considered, and the two protocols will choose the same route otherwise.

Recently the routing optimization was analyzed by using a probabilistic link model [9], which points out that the broadcasting nature of wireless communications should be utilized by routing protocols to achieve robustness at the networking level. Recall that cooperative transmission has been recognized as an effective technology to utilize such feature of wireless communications [5], [10], [11]. Inspired by this observation, we focus on studying the effects of cooperative transmission on the routing decision in this paper. The effects of cooperative transmission to the link quality are first studied. The problem of routing optimization is then investigated in order to study the impact of the improved link cost to the routing decision. The objective function of interest is to minimize the total power consumption with a given end-to-end reliability constraint. The optimal solution of this optimization problem indicates the minimum total power consumption of a route in order to satisfy the required error performance, which is then used as a criterion to compare and select the best among different routes. Our analytical results show that the route chosen by the cooperative criterion can consume much less transmission power compared with the route using only direct transmission. For certain path loss factors and provided that direct transmission is used only, it is observed that a source node will prefer to communicate with its destination node directly, which could result in transmission power higher than the saturation level due to the long 
source-destination distance. However, it is noticeable that the cooperative criterion still maintains the preference of multihop transmission to ensure the transmission power at each link is below the given maximum limit.

\section{System ARChitecture}

In this paper, we consider a wireless ad-hoc network where nodes are assumed to be uniformly distributed in a region with the node density $\lambda$. Each node is equipped with one omnidirectional antenna element (although our results can be extended to more generalized cases with multiple antennas at each node). Time Division Multiple Access (TDMA) schemes are used here for two reasons. First, in case that there are multiple source-destination pairs communicating simultaneously, the TDMA assumption could allow us to only concentrate on one pair, and hence co-channel interference is eliminated automatically. Second, the fact that time division duplex channels are reciprocal naturally makes channel state information available at the transmitter. We employ a propagation model to consider path loss, shadow fading and Rayleigh fading [1], [7], [12]. Denote $g_{i j}$ as the wireless channel coefficient between the nodes $i$ and $j$ and can be modelled as

$$
g_{i j}=\frac{h_{i j}}{d_{i j}^{k / 2}}
$$

where $d_{i j}$ is the distance between the nodes $i$ and $j, 1 / d_{i j}^{k / 2}$ depicts the large-scale behavior of the channel gain, $k$ is the path loss exponent and $h_{i j}$ captures the channel fading characteristics due to the rich scattering environment. Furthermore, multiple nodes are separated apart enough to assume channel fading $h_{i j}$ independent and identically distributed (i.i.d.), complex Gaussian variables with zero mean and unit variance.

Traditionally, the node $i$ communicates directly with the node $j$ in the physical layer, provided that the link $i \rightarrow j$ is delegated by the routing protocol. In this paper, we propose that each node $i$ tries to find one and only one useful relay node (other than the node $j$ ) to accomplish cooperative transmission when communicating with the node $j$. Since cooperative transmission can improve the quality of the wireless link, the existing routing protocols should be modified to take such effect into account. In order to design such a cross-layer routing protocol, it is important to first understand the kind of effects the physical layer technique can bring to the link quality, which will be discussed in detail in the next section.

\section{LINK COST USING COOPERATIVE TRANSMISSION}

This section is to understand how much the quality of wireless links can be improved by using cooperative transmission and what kind of physical characteristics will have the critical effects to the quality improvement. Consider a three-node scenario with nodes $i$ and $j$, and a relay node $R$, where the node $i$ wants to communicate with the node $j$ node with help of the relay node $R$. Such cooperative transmission consists of two stages in time [10]. At the first stage, the node $i$ transmits its information where both the nodes $j$ and $R$ are receiving. At the second stage, the relay decodes and forwards the information of the node $i$ to the node $j$ if decoding is successful. Otherwise the relay will keep silent and the node $i$ will transmit its information once again. For TDMA schemes, such cooperative transmission consumes two times the time slots compared with direct transmission, so data rate at each stage (or time slot) is chosen to be two times of that for direct transmission.

Define $d_{i j}, d_{i R}$, and $d_{R j}$ as the distances among the node $i$, the relay node, and the node $j$. So during the first time slot, the node $j$ receives

$$
y_{j, 1}=\frac{h_{i j}}{d_{i j}^{k / 2}} s_{i}+n_{j, 1}
$$

where $s_{i}$ is the information of the node $i$ and $n_{j}$ will be the white noise. And during the second time slot, the node $j$ receives

$$
y_{j, 2}=\left\{\begin{array}{llll}
\frac{h_{i j}}{d_{i j}^{k / 2}} s_{i}+n_{j, 2} & \text { if } & \left|\frac{h_{R i}}{d_{R i}^{k / 2}}\right|^{2}<q(\rho) \\
\frac{h_{R j}}{d_{R j}^{k / 2}} s_{i}+n_{j, 2} & \text { if } & \left|\frac{h_{R i}}{d_{R i}^{k / 2}}\right|^{2} \geq q(\rho)
\end{array}\right.
$$

where $q(\rho)=\frac{2^{2 R}-1}{\rho}$ and $R$ is the data rate in bits/s/Hz. The signal-to-noise ratio (SNR) is defined as $\rho=E_{b} / N_{0}$ where $E_{b}$ denotes transmission energy per-bit and $N_{0}$ is the one-sided power spectral density of the white noise. As can be seen from (3), the relay node could yield no performance gain if it has a poor link with the source node $i$, which means that the choice of the relay node could be critical to the system performance. For the reason of simplicity, the random choice of relaying is considered in this paper, and the results for using distributed relay selection can be found in [13].

\section{A. Random Choice of Relaying}

Consider that a relay node is selected randomly. Hence the data rate such cooperative system is able to support can be shown as [10]

$\mathcal{I}_{i j}=\left\{\begin{array}{lll}\frac{1}{2} \log \left(1+2 \rho\left|\frac{h_{i j}}{d_{i j}^{k / 2}}\right|^{2}\right) & \text { if } & \left|\frac{h_{R i}}{d_{R i}^{k / 2}}\right|^{2}<q(\rho) \\ \frac{1}{2} \log \left(1+\rho\left[\left|\frac{h_{R j}}{d_{R j}^{k / 2}}\right|^{2}+\left|\frac{h_{i j}}{d_{i j}^{k / 2}}\right|^{2}\right]\right) & \text { if } & \left|\frac{h_{R i}}{d_{R i}^{k / 2}}\right|^{2} \geq q(\rho)\end{array}\right.$

By following the steps in [10], [11], [14], [15] the outage probability is also used here to evaluate error performance. Since $h_{i j}$ is assumed complex Gaussian variables with zero mean and unit variance, $\left|\frac{h_{i j}}{d_{i j}^{k / 2}}\right|^{2}$ is an exponentially distributed variable with parameter $\frac{1}{d_{i j}^{2}}$. So using the results in [10] directly, we have the outage probability between the nodes $i$ and $j$ as

$$
\begin{aligned}
P_{i j}^{C T} & =P\left(\mathcal{I}_{i j}<R\right) \\
& \approx \frac{1}{2} d_{i j}^{k}\left(d_{i R}^{k}+d_{R j}^{k}\right) \frac{\left(2^{2 R}-1\right)^{2}}{\rho^{2}} \\
& =P_{i j}^{d r}\left[\frac{\left(d_{i R}^{k}+d_{R j}^{k}\right)\left(2^{R}-1\right)\left(2^{R}+1\right)^{2}}{2 \rho}\right]
\end{aligned}
$$


where $R$ is the data rate in bit/s/Hz defined by the QoS requirement and the outage probability of direct transmission between the nodes $i$ and $j$ is

$$
P_{i j}^{D T} \approx d_{i j}^{2} \frac{\left(2^{R}-1\right)}{\rho}
$$

Comparing (5) and (6), the necessary condition to ensure cooperative transmission is better than direct transmission is

$$
\left[\frac{\left(d_{i R}^{k}+d_{R j}^{k}\right)\left(2^{R}-1\right)\left(2^{R}+1\right)^{2}}{2 \rho}\right] \leq 1
$$

Another scheme of interest is the traditional two-hop transmission scheme where the destination can only receive signals from the relay node. The outage probability for such two-hop transmission is

$$
\begin{aligned}
P_{i j}^{T H} & =1-\left(1-P_{i R}^{D T}\right)\left(1-P_{R j}^{D T}\right) \\
& \approx d_{i R}^{k} \frac{\left(2^{R}-1\right)}{\rho}+\left(1-d_{i R}^{k} \frac{\left(2^{R}-1\right)}{\rho}\right) d_{R j}^{k} \frac{\left(2^{R}-1\right)}{\rho}
\end{aligned}
$$

where the approximation follows from (6). Comparing (6) and (4), it is evident that the use of cooperative transmission can increase the quality of wireless links in terms of reliability or transmission power consumption. And the use of more advanced cooperative protocols such as in [16], [17] shall provide more performance gain, however, such a simple decodeforward protocol will be used later because of its simplicity.

\section{Route Optimization And Protocol Design}

Previous developed results show that cooperative transmission can bring some performance gain to the physical layer, specially to the quality of wireless links. It is of interest to study how such such physical layer benefits can have effects to the upper layer, such as the routing protocol design.

Consider that a route has been constructed between the source and destination. The goal of interest here is to understand the best performance a random route can achieve, which can be later used as a quantitative criterion for route selecting. Without losing generality, the nodes sitting on the route are denoted as $S \rightarrow 1 \ldots \rightarrow n \rightarrow D$. Different to traditional routes, cooperative transmission is used to improve the link quality when the node $i$ is communicating with the node $j$. It is possible that a good helping node is not available for some pairs of the $n+1$ links of the route. In that case, direct transmission is used instead of relying on cooperative transmission. Hence the $n+1$ links can be categorized into two sets. One set, defined as $\mathcal{S}_{1}$, includes all links using cooperative transmission and the other one, defined as $\mathcal{S}_{2}$, includes the links using direct transmission. Note that $\left|\mathcal{S}_{1}\right|+\left|\mathcal{S}_{2}\right|=n+1$ since there are only $n+1$ links on the route.

Provided that the $i \rightarrow j$ link utilizes cooperative transmission, $i j \in \mathcal{S}_{1}$, define $\rho_{i j}=f_{C T}^{-1}\left(P_{i j}^{C T}\right)$ where $\rho_{i j}$ is the required SNR for the link from the node $i$ node to the node $j$, and

$$
f_{C T}^{-1}(x)=\left(2^{2 R}-1\right) \sqrt{\frac{d_{i j}^{k}\left(d_{i R}^{k}+d_{R j}^{k}\right)}{2 x}} .
$$

Hence the transmission power for the $i \rightarrow j$ link is $W_{i j}=$ $2 R B N_{0} \rho=2 R B N_{0} f_{C T}^{-1}\left(P_{i j}^{C T}\right)$. If $i j \in \mathcal{S}_{2}$, the transmission power for for the $i \rightarrow j$ link is $W_{i j}=R B N_{0} \rho=$ $R B N_{0} f_{D T}^{-1}\left(P_{i j}^{D T}\right)$, where

$$
f_{D T}^{-1}(x)=\left(2^{R}-1\right) \frac{d_{i j}^{k}}{x} .
$$

The problem to minimize the total transmission power consumption with the constraint on the end-to-end reliability can be formulated as

$$
\begin{array}{ll}
\min _{P_{i j}^{D T}, P_{i j}^{C T}} & \sum_{i j \in \mathcal{S}_{1}} W_{i j}+\sum_{i j \in \mathcal{S}_{2}} W_{i j} \\
\text { s.t. } & 1-\prod_{i j \in \mathcal{S}_{1}}\left(1-P_{i j}^{C T}\right) \prod_{i j \in \mathcal{S}_{2}}\left(1-P_{i j}^{D T}\right) \leq P
\end{array}
$$

For small outage probability $P_{i j}^{D T}<<1$ and $P_{i j}^{C T}<<1$, we can have the following approximation

$$
\begin{array}{r}
1-\prod_{i j \in \mathcal{S}_{1}}\left(1-P_{i j}^{C T}\right) \prod_{i j \in \mathcal{S}_{2}}\left(1-P_{i j}^{C T}\right) \\
\approx \sum_{i j \in \mathcal{S}_{1}} P_{i j}^{C T}+\sum_{i j \in \mathcal{S}_{2}} P_{i j}^{D T} .
\end{array}
$$

So the optimization problem can be simplified as

$$
\begin{array}{ll}
\min _{P_{i j}^{D T}, P_{i j}^{C T}} & \sum_{i j \in \mathcal{S}_{1}} W_{i j}+\sum_{i j \in \mathcal{S}_{2}} W_{i j} \\
\text { s.t. } & \sum_{i j \in \mathcal{S}_{1}} P_{i j}^{C T}+\sum_{i j \in \mathcal{S}_{2}} P_{i j}^{D T} \leq P
\end{array}
$$

By introducing an auxiliary variable $z,(12)$ can be written as

$$
\begin{array}{ll}
\min _{P_{i j}^{D T}, P_{i j}^{C T}, z} & \sum_{i j \in \mathcal{S}_{1}} W_{i j}+\sum_{i j \in \mathcal{S}_{2}} W_{i j} \\
\text { s.t. } & \sum_{i j \in \mathcal{S}_{1}} P_{i j}^{C T} \leq P-z \\
& \sum_{i j \in \mathcal{S}_{2}} P_{i j}^{D T} \leq z \\
& 0 \leq z \leq P
\end{array}
$$

Note that the transmission power is always positive, $W_{i j} \geq$ 0 , and both $f_{C T}^{-1}(x)$ and $f_{D T}^{-1}(x)$ are monotonic decreasing. Hence the optimization can be solved in two stages. First we treat $z$ as a constant and solve the following two optimization problems separately,

$$
\begin{array}{llll}
\min _{P_{i j}^{C T}} & \sum_{i j \in \mathcal{S}_{1}} W_{i j} & \min _{P_{i j}^{D T}} & \sum_{i j \in \mathcal{S}_{2}} W_{i j} \\
\text { s.t. } & \sum_{i j \in \mathcal{S}_{1}} P_{i j}^{C T} \leq P-z & \text { s.t. } & \sum_{i j \in \mathcal{S}_{2}} P_{i j}^{D T} \leq z
\end{array}
$$


which yields the two solutions

$$
\begin{aligned}
\sum_{i j \in \mathcal{S}_{1}} W_{i j}= & 2 R B N_{0}\left(\frac{2^{2 R}-1}{\sqrt{2(P-z)}}\right) \\
& \times\left(\sum_{i j \in \mathcal{S}_{1}}\left[d_{i j}^{k}\left(d_{i R}^{k}+d_{R j}^{k}\right)\right]^{\frac{1}{3}}\right)^{\frac{3}{2}} \\
\sum_{i j \in \mathcal{S}_{2}} W_{i j}= & R B N_{0} \frac{\left(2^{R}-1\right)\left(\sum_{i j \in \mathcal{S}} d_{i j}^{\frac{k}{2}}\right)^{2}}{z}
\end{aligned}
$$

The results in (14) and (15) can be obtained by applying the Kuhn-Tucker condition in [18]. Due to the space limitation, the details of the development for the optimization is omitted here and will be provided in in [13]. Note that both $\sum_{i j \in \mathcal{S}_{1}} W_{i j}$ and $\sum_{i j \in \mathcal{S}_{2}} W_{i j}$ now becomes functions of the auxiliary variable $z$.

The second step is solve the following optimization

$$
\begin{aligned}
& \min _{z} \quad f_{z}(z)=R B N_{0} \frac{\left(2^{R}-1\right)\left(\sum_{i j \in \mathcal{S}} d_{i j}^{\frac{k}{2}}\right)^{2}}{z} \\
& +2 R B N_{0}\left(\frac{2^{2 R}-1}{\sqrt{2(P-z)}}\right)\left(\sum_{i j \in \mathcal{S}_{1}}\left[d_{i j}^{k}\left(d_{i R}^{k}+d_{R j}^{k}\right)\right]^{\frac{1}{3}}\right)^{\frac{3}{2}}
\end{aligned}
$$$$
\text { s.t. } \quad 0 \leq z \leq P
$$

which is difficult to solve directly as it could result in a equation with degree higher than 2 . Note that $f_{z}(z)$ is a strictly convex function for $0 \leq z \leq P$ since $\frac{d^{2} f_{z}(z)}{d^{2} z} \leq 0$. Hence there will only one minimum for $0 \leq z \leq P$, defined as $z^{*}$. Provided that $\left.\frac{d f_{z}(z)}{d z}\right|_{z=\frac{P}{2}} \geq 0$, it can be expected that $0 \leq z^{*} \leq \frac{P}{2}$, otherwise $\frac{P}{2} \stackrel{\frac{2}{2}}{\leq} z^{*} \leq P$. So in the following, the close form of $z^{*}$ will be shown as

$$
z^{*}=\left\{\begin{array}{lll}
\frac{2 P \sqrt{z_{1}}}{\sqrt{z_{1}}+\sqrt{z_{2}}} & \text { if } & \left.\frac{d f_{z}(z)}{d z}\right|_{z=\frac{P}{2}} \geq 0 \\
P-\left(\frac{z_{3}}{2 z_{4}}\right)^{2 / 3} & \text { if } & \left.\frac{d f_{z}(z)}{d z}\right|_{z=\frac{P}{2}}<0
\end{array}\right.
$$

and the total power consumption is

$$
W=\left\{\begin{array}{lll}
\frac{\left(\sqrt{z_{1}}+\sqrt{z_{2}}\right)^{2}}{2 P} & \text { if } & \left.\frac{d f_{z}(z)}{d z}\right|_{z=\frac{P}{2}} \geq 0 \\
\left(2 z_{4}\right)^{1 / 3} z_{3}^{2 / 3}+\frac{z_{4}(P)^{2}}{P-\left(\frac{z_{3}}{2 z_{4}}\right)^{2 / 3}} & \text { if } & \left.\frac{d f_{z}(z)}{d z}\right|_{z=\frac{P}{2}}<0
\end{array}\right.
$$

where $z_{1}=R B N_{0}\left(2^{R}-1\right)\left(\sum_{i j \in \mathcal{S}_{2}} d_{i j}^{\frac{k}{2}}\right)^{2}, z_{2}=$ $2 R B N_{0} \sqrt{2 P}\left(2^{2 R}-1\right)\left(\sum_{i j \in \mathcal{S}_{1}}\left[d_{i j}^{k}\left(d_{i R}^{k}+d_{R j}^{k}\right)\right]^{\frac{1}{3}}\right)^{\frac{3}{2}}$, $z_{3}=2 R B N_{0}\left(\frac{2^{2 R}-1}{\sqrt{2}}\right)\left(\sum_{i j \in \mathcal{S}_{1}}\left[d_{i j}^{k}\left(d_{i R}^{k}+d_{R j}^{k}\right)\right]^{\frac{1}{3}}\right)^{\frac{3}{2}}$ and $z_{4}=R B N_{0} \frac{\left(2^{R}-1\right)\left(\sum_{i j \in \mathcal{S}} d_{i j}^{\frac{k}{2}}\right)^{2}}{(P)^{2}}$. The details of the derivation for (17) can be from the [13].

1) A Special Case: Consider the worst case where each $i \rightarrow j$ link can not find a good relay node satisfy (7). Then all links will use direct transmission, and hence the total power consumption will be

$$
\begin{aligned}
W & =z_{4} P \\
& =\frac{R B N_{0}\left(2^{R}-1\right)\left(\sum_{i j \in \mathcal{S}_{2}} d_{i j}^{\frac{k}{2}}\right)^{2}}{P} .
\end{aligned}
$$

In the case of $k=2$, it is interesting to find that

$$
W=\frac{R B N_{0}\left(2^{R}-1\right)\left(\sum_{i j \in \mathcal{S}_{2}} d_{i j}\right)^{2}}{P} .
$$

For the two successive links $i \rightarrow j \rightarrow l$. we can have $d_{i j}+d_{j l} \geq d_{i l}$ since they are three edges of a triangle. Hence the one-hop transmission from the source to the destination directly would be preferred by the criterion in (20).

2) Route Selection: For more general cases, the quality of one route will be determined jointly by the cooperative links as well as the direct-transmission links. Two rules for routing decision have been implied by the developed results. The preference of the cooperative links can be illustrated from the object function in (16). The power consumed by cooperative links is inverse proportional to the square of the outage probability whereas the power consumed by direct transmission is inverse proportional to the probability. Provided that both $z^{*}$ and $P$ are very small and at the same order, it can be expected that replacing a direct-transmission link with a cooperative link can reduce the power consumption. The preference for multiple-hop transmission can be illustrated by the following example.

Consider a route where each of its links, $i \rightarrow j$, can find a good-quality helping node in the middle of the straight line between the nodes $i$ and $j$. With such assumption, the total transmission power consumed by the route only using one hop can be written as

$$
W_{\text {onehop }}=2 R B N_{0}\left(\frac{2^{2 R}-1}{\sqrt{2 P}}\right) \frac{d^{2}}{\sqrt{2}}=\frac{d^{2}\left(2^{2 R}-1\right)}{2 \sqrt{P}}(21)
$$

and the power consumption for the route using $n$-hop transmission can be shown as

$$
\begin{aligned}
W_{\text {nhop }} & =2 R B N_{0}\left(\frac{2^{2 R}-1}{\sqrt{2 P}}\right)\left(n\left[\frac{d^{2}}{n^{2}} \frac{d^{2}}{2 n^{2}}\right]^{\frac{1}{3}}\right)^{\frac{3}{2}} \\
& =2 R B N_{0} \frac{n^{\frac{3}{2}} d^{2}\left(2^{2 R}-1\right)}{2 n^{2} \sqrt{P}}=\frac{1}{n^{1 / 2}} W_{\text {onehop }}, n \geq 2,
\end{aligned}
$$

which shows that the use of multiple-hop transmission can reduce the transmission power consumption.

3) Numerical Results: In the following, we provide an example to show the effect of the criterion of minimizing the total transmission power with the constraint of the end-to-end reliability on the routing decision. The required data rate is $R=0.1 \mathrm{bit} / \mathrm{s} / \mathrm{Hz}$ and $d_{S R}=10 \mathrm{~m}$. In the first setup, there are two intermediate nodes sitting on the straight line between the source and destination with $d_{S 1}=d_{2 D}=\frac{1}{3} d_{S D}$. The noncooperative criterion in (20) will pick up the route $S \rightarrow D$ since there is no point to use multiple-hop transmission which yields the same power consumption, but more delay. However 


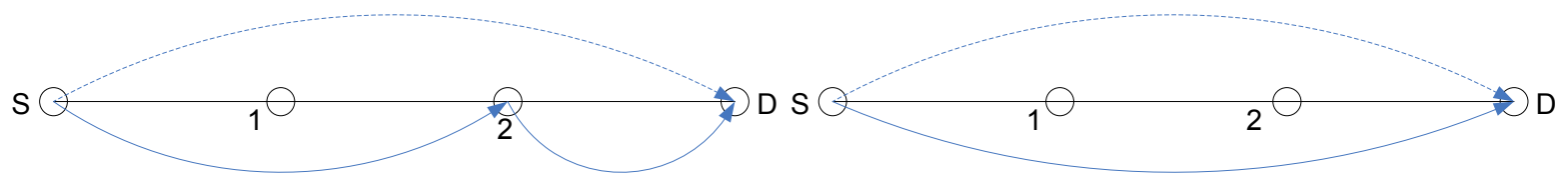

(a) Setup 1: Two intermediate nodes for $P>0.043$

(b) Setup 1: Two intermediate nodes for $P \leq 0.043$

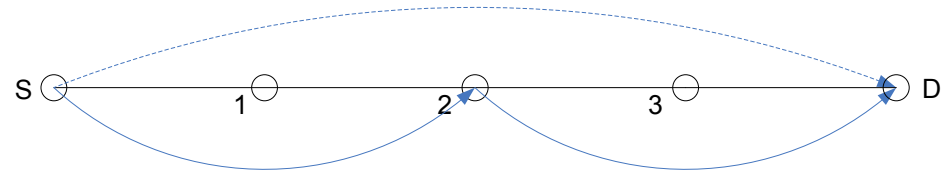

(c) Setup 2: Three intermediate nodes for all $P$

Fig. 1. Route selection with or without cooperative transmission (CT). The data rate is set as $R=0.1 \mathrm{bit} / \mathrm{s} / \mathrm{Hz}$, and the source-destination distance is $10 \mathrm{~m}$. The solid line presents the route which will be chosen by the criterion in (18), and the dash line presents the route chosen by the criterion without cooperative transmission $\left(\left|\mathcal{S}_{1}\right|=0\right)$.

for the criterion proposed in (18), the route, $S \Rightarrow 2 \rightarrow D$, will be chosen for the mild requirement of the end-to-end reliability, where $\Rightarrow$ denotes a link using cooperative transmission. For the highly demanding reliability requirement, the error probability could be so small that the power consumed by the direct-transmission link $2 \rightarrow D$ will dominate the power consumption, which results the route $S \Rightarrow D$ as the preferred one. In Table I shows that these routes picked by the proposed criterion can reduce the power consumption significantly compared with the scheme only using direct transmission. For the second setup, there are three intermediate nodes sitting on the straight line between the source and destination with $d_{S 1}=d_{2 D}=\frac{1}{4} d_{S D}$. The situation is much easier where the route with multi-hop transmission will be chosen, and its performance is also shown in the Table I.

TABLE I

NORMALIZED TRANSMIT POWER CONSUMPTION CHOSEN BY TWO CRITERIA

\begin{tabular}{|l|l|l|l|l|l|}
\hline$P$ & 0.1 & 0.05 & 0.01 & 0.005 & 0.001 \\
\hline Direct trans., $S \rightarrow D$ & 71.773 & 143.55 & 717.73 & 1435.5 & 7177.3 \\
Setup 1, $S \Rightarrow 2 \rightarrow D$ & 43.144 & 68.235 & 268.18 & 426.11 & 1486.2 \\
Setup 1, $S \Rightarrow D$ & 49.566 & 70.097 & 156.74 & 221.67 & 495.66 \\
Setup 2, $S \Rightarrow 2 \Rightarrow D$ & 33.25 & 47.023 & 105.15 & 148.7 & 332.5 \\
\hline
\end{tabular}

\section{CONCLUSION}

In this paper, we have studied the impact of cooperative transmission on the routing decision for wireless ad-hoc networks. The influence of cooperative transmission to the quality of wireless links is discussed first at the physical layer. The objective function of interest is to minimize the total power consumption with the constraint of the end-to-end reliability. Both analytical and simulation results are provided to show that the criteria using cooperative transmission typically yield more efficient routes than the non-cooperative schemes. At the current stage, only random choice of relays has been used, and it is interesting to study cross-layer routing using more advanced cooperative protocols, such as the best-relay scheme and distributed beamforming. Furthermore, a centralized scheme is required for the proposed routing selection, and hence it will be an interesting future topic to design a distributed routing protocol with the use of the developed quantitative criterion.

\section{REFERENCES}

[1] C.-J. Chen and L.-C. Wang, "Enhancing coverage and capacity for multiuser MIMO system by utilizing scheduling," IEEE Trans. on Wireless Communications, vol. 5, pp. 1148-1157, May 2006.

[2] T. Yoo and A. Goldsmith, "On the optimality of multiantenna broadcast scheduling using zero-forcing beamforming," IEEE Journal on Select. Areas in Comm., vol. 24, pp. 528-540, Mar. 2006.

[3] G. Foschini and M. Gans, "On limits of wireless communication in a fading environment when using multiple antennas," Wireless Personal Communications, vol. 6, no. 3, pp. 311-335, Mar. 1998.

[4] E. Telatar, "Capacity of multi-antenna gaussian channels," European Trans. Telecom., vol. 10, pp. 585-595, Nov./Dec. 1999.

[5] J. N. Laneman and G. W. Wornell, "Distributed space-time-coded protocols for exploiting cooperative diversity in wireless networks," IEEE Trans. Information Theory, vol. 49, pp. 2415-2425, Oct. 2003.

[6] A. Sendonaris, E. Erkip, and B. Aazhang, "User cooperation diversitypart I: system description," IEEE Trans. communications, vol. 51, pp. 1927-1938, Nov. 2003.

[7] S. Cui and A. J. Goldsmith, "Cross-layer design in energy-constrained networks using cooperative MIMO techniques," EURASIP/Elsevier Signal Processing Journal, vol. 86, pp. 425-436, Aug. 2006.

[8] S. Cui, A. J. Goldsmith, and A. Bahai, "Energy-constrained modulation optimization," IEEE Transactions on Wireless Communications, vol. 4, pp. 2349-2360, Sept. 2005.

[9] E. Khandani, E. Modiano, J. Abounadi, and L. Zheng, "Reliability and route diversity in wireless networks," in Proc. Conf. on Information Sceiences and Systems, The Johns Hopkins University, USA, Mar 2005.

[10] J. N. Laneman, D. N. C. Tse, and G. W. Wornell, "Cooperative diversity in wireless networks: Efficient protocols and outage behavior," IEEE Trans. Information Theory, vol. 50, pp. 3062-3080, Dec. 2004.

[11] Z. Ding, T. Ratnarajah, and C. Cowan, "On the diversity-multiplexing tradeoff for wireless cooperative multiple access systems," IEEE Trans. Signal Processing, pp. 4627-4638, Sept. 2007.

[12] J. Proakis, Digital Communications, 4th ed. McGraw-Hill, New York, 2000.

[13] Z. Ding and K. K. Leung, "On routing decision by using cooperative transmission in wireless ad-hoc networks," IEEE Trans. Vechicular Technology, (submitted.).

[14] L. Zheng and D. N. C. Tse, "Diversity and multiplexing : a fundamental tradeoff in multiple antenna channels," IEEE Trans. Information Theory, vol. 49, pp. 1073-1096, May 2003.

[15] D. N. C. Tse, P. Viswanath, and L. Zheng, "Diversity-multiplexing tradeoff in multiple-access channels," IEEE Trans. Information Theory, vol. 50, pp. 1859-1874, Sept. 2004.

[16] Z. Ding, Y. Gong, T. Ratnarajah, and C. Cowan, "On the performance of opportunistic cooperative wireless networks," IEEE Transactions on Communications, (accepted).

[17] Z. Ding, W. Chen, and K. Leung, "Distributed beamforming and power allocation for cooperative networks," IEEE Transactions on Wireless Communications, (accepted).

[18] S. Boyd and L. Vandenberghe, Convex optimization. Cambridge University Press, 2003. 